\title{
Relación entre personalidad oscura, consumo de sustancias y felicidad
}

\author{
Benito Nohales Nieto \\ beninohales21@gmail.com \\ LAURA MEZQUITA GUILLAMÓN \\ Imezquit@uji.es \\ MANUEL IGNACIO IBÁÑEZ RIBES \\ iribes@uji.es
}

\section{Resumen}

Introducción: La triada oscura divide la personalidad socialmente aversiva en tres dimensiones: maquiavelismo, narcisismo subclínico y psicopatía subclínica. Los instrumentos más utilizados para evaluarla de forma conjunta son el Dirty Dozen (DD) y el Short Dark Triad (SD3), que recientemente han sido adaptados a nuestro contexto sociocultural. Este estudio pretende examinar la red nomológica de estas escalas y cómo se relacionan con el consumo de sustancias (nicotina, alcohol y cannabis) y la felicidad subjetiva. Método: Una muestra de 217 sujetos cumplimentaron a través de internet nuestras versiones en español del DD y SD3, la escala AIS de consumo de sustancias y la escala de felicidad subjetiva. Resultados: Los resultados muestran que el maquiavelismo no se relaciona con ninguna sustancia en la regresión pero en las escalas se correlaciona con consumo de alcohol; el narcisismo se relaciona con el consumo de alcohol y cannabis, además el narcisismo SD3 con la felicidad; la psicopatía se relaciona con todas las variables de consumo, pero la psicopatía DD no tiene relación con el tabaco. Conclusión: En definitiva, y de acuerdo con lo esperado, la psicopatía sería la faceta que más se relaciona con el consumo de sustancias seguida del narcisismo; esta última es además la más relevante para el bienestar subjetivo. No obstante, los resultados obtenidos por ambos instrumentos muestran algunas diferencias, observando que el SD3 se ajusta mejor a lo esperado. Por ello, concluimos que la adaptación a nuestro contexto sociocultural del SD3 mostraría una mayor validez de constructo en la evaluación de la triada oscura que el DD.

Palabras clave: triada oscura, maquiavelismo, narcisismo, psicopatía, consumo de sustancias.

\section{Abstract}

Introduction: The Dark Triad divides socially aversive personality into three traits: Machiavellianism, subclinical narcissism and subclinical psychopathy. The most widely used instruments to measure the three traits together are Dirty Dozen (DD) and the Short Dark 
Triad (SD3), which have recently been adapted to our socio-cultural context. This study aims to examine the nomological network of these scales, and how they relate to substance use (nicotine, alcohol and cannabis) and subjective happiness. Method: A sample of 217 participants completed our Spanish versions of DD and SD3, AIS Scale of Substance Use, and Subjective Happiness Scale online. Results: The results showed that: Machiavellianism is not related to any substance use in regression, but correlates with alcohol use on scales; narcissism is related to alcohol and cannabis use; SD3 narcissism correlates with happiness; psychopathy is related to all the substance use variables, DD psychopathy does not correlate with tobacco. Conclusion: Definitely, and as expected, psychopathy would be the most related triad aspect to substance use, followed by narcissism, and the latter would also be the most relevant to subjective well-being. However, the results obtained from both instruments showed some differences. We observed that SD3 better matched expectations. So we conclude that the adaptation of SD3 to our socio-cultural context would show higher construct validity in the Dark Triad evaluation than DD.

Keywords: Dark Triad, Machiavellianism, narcissism, psychopathy, substance use.

\section{Introducción}

Maquiavelismo, narcisismo y psicopatía son los tres rasgos de personalidad que componen la que se conoce como la triada oscura de la personalidad, también conocida como triope. El concepto original es dark triad of personality, adoptado por Paulhus y Williams en el año 2002 en el artículo «The Dark Triad of Personality: Narcissism, Machiavellianism, and Psychopathy» (Paulhus y Williams, 2002)

para referirse a las tres grandes dimensiones de personalidad consideradas como dañinas o destructivas para las personas con las que interactúan los individuos con alta puntuación en estas dimensiones. Cabe destacar que el modelo se refiere a estos factores como características normales de personalidad que se pueden encontrar en la población general y con un nivel subclínico, es decir, que no tienen por qué suponer una patología para el individuo. Así pues, los términos exactos de sus constructos son maquiavelismo, narcisismo subclínico (diferenciar del narcisismo clínico del trastorno narcisista de la personalidad) y psicopatía subclínica (diferenciar de la psicopatía clínica). Sin embargo, nos referimos a ellos en todo el estudio como maquiavelismo, narcisismo y psicopatía. Estos son el significado y las características de cada constructo:

- Maquiavelismo: manipulación y explotación de otras personas para conseguir los propios fines; se refiere tanto a la capacidad de manipular realizando planes a largo plazo como a obtener satisfacción con esta manipulación. En un estudio reciente (Nohales, 2015) mediante análisis factorial, este constructo se podía diferenciar en dos tipos: maquiavelismo manipulador (más directo para conseguir un beneficio inmediato) y maquiavelismo estratégico (intentar controlar diversos aspectos sociales como la reputación o la información que puedan ser útiles con el objetivo de obtener de las personas un beneficio propio cuando sea preciso).

- Narcisismo: grandiosidad o admiración excesiva hacia uno mismo, querer la admiración del resto, creerte superior a las demás personas y que por ello mereces un trato especial. 
- Psicopatía: baja empatía, impulsividad, conducta antisocial, insensibilidad hacia los sentimientos de los demás y ausencia o poco remordimiento sobre las conductas propias que dañan a otros, lo que implica poca o falta de moral en las acciones propias.

Existen diferentes instrumentos para evaluar las dimensiones de la triada de forma específica. No obstante, y con el fin de facilitar la investigación en este campo, en los últimos años se han desarrollado cuestionarios breves que permitan medir las tres dimensiones simultáneamente. Los dos principales instrumentos que sirven para medir los tres constructos en un mismo cuestionario y que han demostrado ser fiables y válidos son el Dirty Dozen de Jonason y Webster (2010) y el Short Dark Triad de Jones y Paulhus (2014), que recientemente hemos adaptado a nuestro contexto sociocultural (Nohales, 2015).

Los rasgos que componen la triada oscura han mostrado su relevancia en diferentes ámbitos de la vida, especialmente aquellos asociados a comportamientos más problemáticos, como la conducta antinormativa o la delincuencia (Chabrol, Van Leeuwen, Rodgers y Séjourné, 2009). Un aspecto fuertemente asociado al comportamiento antinormativo es el consumo de sustancias, especialmente abusivo. Aunque la investigación al respecto es todavía escasa, según los estudios realizados hasta el momento parece claro que el rasgo de psicopatía estaría moderadamente asociado a un mayor consumo (Chabrol y cols., 2009; Jonason, Koenig y Tost, 2010; Jonason, Baughman, Carter y Parker, 2015). En relación al narcisismo y maquiavelismo, los resultados son menos consistentes. Así, todos estos estudios encuentran relación entre la psicopatía y el consumo de diferentes sustancias. Jonason y cols. (2010) y Chabrol y cols. (2009) encuentran relación entre maquiavelismo y narcisismo con consumo de alcohol, pero estos resultados no se observan en el estudio de Jonason y cols. (2015). Además, en este último se encuentra relación entre la psicopatía y el narcisismo con el consumo de riesgo.

Por otra parte, los aspectos positivos de estas características de personalidad han sido poco explorados. Que conozcamos, tan solo unos pocos estudios han investigado la relación entre rasgos de la triada oscura y aspectos psicológicos beneficiosos como la felicidad o la autoestima. Concretamente, en el artículo «The Dark Triad, happiness and subjective wellbeing» (Egan, Chan y Shorter, 2014)

se encontró una correlación positiva entre el narcisismo y el bienestar subjetivo, mientras que otro estudio encontró relación entre el narcisismo y la autoestima (Raskin, Novacek y Hogan, 1991). Por otra parte, en el estudio citado anteriormente de Jonason y cols. (2015) que pretende medir la relación de la triada con más variables beneficiosas, se observa una relación positiva del narcisismo con autoestima, esperanza, bienestar emocional, bienestar psicológico y bienestar social.

El objetivo de este estudio es examinar la red nomológica de las escalas del DD y SD3 y saber cómo se relacionan las dimensiones de la triada oscura con el consumo de diferentes sustancias (alcohol, tabaco y cannabis) y con la felicidad subjetiva. Esto puede ser importante ya que la identificación de perfiles de personalidad propensos al abuso de drogas puede ser útil para su prevención.

De este modo, basándonos en los estudios citados anteriormente, las hipótesis del estudio son que el rasgo de la triada que más se relacionará con el consumo de sustancias será la psicopatía, seguida del narcisismo, y que se encontrará relación postiva entre el narcisismo y la felicidad subjetiva. 


\section{Método}

\section{Muestra}

La muestra que cumplimentó telemáticamente todos los cuestionarios fue de 215 sujetos $(\mathrm{N}=215)$. De ellos, 146 son mujeres $(67,9 \%)$ y 69 son hombres $(32,1 \%)$, la edad media de estos participantes se sitúa en 25,28 años (desviación típica = 7,67), teniendo sujetos desde los 18 hasta los 63 años.

\section{Instrumentos}

En el estudio se utilizan 4 instrumentos diferentes para medir las dimensiones de la triada oscura, consumo de sustancias y felicidad subjetiva. Los instrumentos son los siguientes:

- Dirty Dozen (DD; Jonason y Webster, 2010) y Short Dark Triad (SD3; Jones y Paulhus, 2014): estos dos instrumentos fueron usados para medir las dimensiones de la triada oscura de forma conjunta. Se utilizaron las versiones que recientemente hemos adaptado a nuestro contexto sociocultural (Nohales, 2015). Ambos instrumentos cuentan con 3 subescalas, una para cada dimensión de la triada, teniendo el DD 4 ítems por subescala (total $=12$ ) y el SD3 9 ítems por subescala (total $=27$ ). Además, los dos usan una escala tipo Likert de 5 opciones (totalmente en desacuerdo - totalmente de acuerdo).

- Escala AIS de consumo de sustancias (Grau y Ortet, 1999): evalúa el consumo por parte de los sujetos de nicotina, alcohol y cannabis entre semana y en fin de semana, además del consumo del mejor amigo, constando con un total de 12 ítems (los ítems de consumo del mejor amigo no se incluyeron finalmente en el estudio). Las respuestas a cada ítem son un valor numérico para indicar cuántos cigarrillos, cervezas o vasos de vino, cubatas o chupitos y porros se consumen. Para calcular los valores totales de consumo de nicotina y cannabis se suman las cantidades de estas sustancias; para calcular el consumo de alcohol cada cerveza o copa de vino es equivalente a 1 unidad de bebida estándar y cada cubata o chupito a 2 unidades de bebida estándar (UBEs; Rodríguez-Martos, Gual y Llopis, 1999).

- Escala de felicidad subjetiva (Lyubomirsky y Lepper, 1999): evalúa la felicidad como bienestar subjetivo, es decir, el grado de felicidad que siente una persona visto desde la perspectiva de aquel que responde. Se compone de 4 ítems con escala Likert de 1 a 7 .

\section{Procedimiento}

Una vez escogidos los instrumentos, se prepararon para que estuvieran disponibles en un enlace web mediante la plataforma Googleforms. Este enlace web con todos los instrumentos fue distribuido por correo electrónico para conseguir la mayor muestra posible. Para llevar a cabo esta distribución, el enlace con los instrumentos se envió a conocidos y se distribuyó por las redes sociales, mientras que los miembros del grupo IDAP enviaron un correo electrónico para pedir colaboración a los alumnos de las asignaturas que imparte cada uno. Además, también se pidió que después de realizar los cuestionarios se enviaran 
a conocidos de los sujetos, con la intención de obtener un efecto bola de nieve que consiguiera más muestra.

\section{Análisis}

Para realizar los análisis estadísticos se utilizó el programa informático SPSS (versión 22.0). Para empezar, se calcularon las correlaciones bivariadas de Pearson para medir la relación de cada subescala de los instrumentos de la triada oscura con la felicidad y el consumo de tabaco, alcohol y cannabis. Seguidamente, se realizó un análisis de regresión lineal múltiple de pasos sucesivos, controlando por edad y sexo para poder saber el efecto directo de las variables de la triada sobre el consumo y la felicidad. En esta regresión se introdujeron, en un primer paso, la edad y el sexo y, en un segundo paso, como variables predictoras, se utilizaron el maquiavelismo manipulador, el maquiavelismo estratégico, el narcisismo y la psicopatía. Estos son los factores de personalidad oscura extraídos recientemente a partir de un análisis factorial conjunto de las escalas DD y SD3 (Nohales, 2015).

\section{Resultados}

Correlaciones bivariadas de Pearson entre DD y SD3 - Consumo de sustancias y felicidad.

A continuación se muestran los resultados entre las correlaciones de las subescalas de los intrumentos de la triada con los de consumo y felicidad; todas las correlaciones obtenidas pueden observarse en la tabla 1.

- Maquiavelismo: en el DD solo muestra correlación significativa con el consumo de alcohol entre semana $\left(r=, 137^{*}\right)$, mientras que en el SD3 con el consumo de alcohol entre semana $\left(r=, 141^{*}\right)$ y el total $\left(r=, 141^{*}\right)$.

- Narcisismo: en el DD muestra correlación significativa con el consumo de alcohol total $\left(r=, 182^{* *}\right)$, en fin de semana $\left(r=, 169^{*}\right)$, el cannabis total $\left(r=, 142^{*}\right)$ y entre semana $\left(r=, 144^{*}\right)$; mientras que en el SD3 con el consumo de cannabis total $\left(r=, 143^{*}\right)$, entre semana $\left(r=, 152^{*}\right)$ y con felicidad $\left(r=, 199^{* *}\right)$.

- Psicopatía: en el DD muestra correlación significativa con el consumo de alcohol total $\left(r=, 145^{*}\right)$, en fin de semana $\left(r=, 148^{*}\right)$, el cannabis total $\left(r=, 137^{*}\right)$ y en fin de semana $\left(r=, 141^{*}\right)$; mientras que en el SD3 la psicopatía muestra correlación significativa con todas la variables de consumo pero no con felicidad.

Tabla 1

Correlaciones bivariadas de Pearson entre DD y SD3 - Sustancias y felicidad

\begin{tabular}{lcccccc}
\hline & DDMaqu & DDNar & DDPsic & SD3Maqu & SD3Nar & SD3Psic \\
\hline Alcohol total &, 121 &, $182^{* *}$ &, $145^{*}$ &, $141^{*}$ &, 110 &, $286^{* *}$ \\
Alcohol entre semana &, $137^{*}$ &, 121 &, 064 &, $141^{*}$ &, 065 &, $255^{\star *}$ \\
Alcohol fin de semana &, 090 &, $169^{* *}$ &, $148^{*}$ &, 112 &, 106 &, $239^{* *}$ \\
\hline Tabaco total &, 075 &, 030 &, 055 &, 085 &, 060 &, $168^{*}$ \\
Tabaco entre semana &, 071 &, 016 &, 042 &, 069 &, 044 &, $150^{*}$
\end{tabular}




\begin{tabular}{lcccccc}
\hline & DDMaqu & DDNar & DDPsic & SD3Maqu & SD3Nar & SD3Psic \\
\hline Tabaco fin de semana &, 079 &, 058 &, 080 &, 114 &, 092 &, $194^{*}$ \\
\hline Cannabis total &, 099 &, $142^{*}$ &, $137^{*}$ &, 120 &, $143^{*}$ &, $237^{* *}$ \\
Cannabis entre semana &, 097 &, $144^{*}$ &, 127 &, 113 &, $152^{*}$ &, $220^{* *}$ \\
Cannabis fin de semana &, 092 &, 127 &, $141^{*}$ &, 121 &, 114 &, $243^{* *}$ \\
\hline Felicidad &, 008 &,- 029 &,- 028 &, 000 &, $199^{* *}$ &,- 034 \\
\hline
\end{tabular}

Nota. ${ }^{* *} p<, 01 ;{ }^{*} p<, 05$

\section{Regresión lineal}

En la tabla 2 se observan los resultados del análisis de regresión lineal entre las variables totales de consumo de sustancias y felicidad con sexo, edad y los factores totales de personalidad oscura. Los coeficientes con alta significación están marcados en negrita y asteriscos mientras que la tendencia está marcada en cursiva y con el símbolo de la suma (+). Controlando por edad y sexo, las variables totales de la triada oscura muestran las siguientes regresiones significativas:

- Maquiavelismo manipulador y maquiavelismo estratégico: ninguno de los dos tipos de maquiavelismo obtiene significación con el consumo total de ninguna sustancia ni con la felicidad.

- Narcisismo: obtiene significación con consumo total de cannabis y muestra tendencia al consumo de alcohol y a la felicidad.

- Psicopatía: obtiene significación con el consumo total de las tres sustancias (nicotina, alcohol y cannabis) pero no muestra ningun tipo de relación con la felicidad.

El paso 2 (las 4 dimensiones de personalidad oscura juntas) influye significativamente en el consumo de las tres sustancias pero no en felicidad. Respecto al sexo, los hombres obtienen coeficientes más altos que las mujeres en el consumo de alcohol y cannabis, pero en el consumo de nicotina no se encuentran diferencias significativas. Además, los más jóvenes muestran mayores puntuaciones en consumo de alcohol, mientras que los participantes de más edad tienden a mostrar un mayor consumo de tabaco. El paso 1 influye significativamente en el consumo de las tres sustancias pero no en la felicidad.

Tabla 2

Análisis de regresión lineal

\begin{tabular}{|c|c|c|c|c|c|c|c|c|c|}
\hline & & \multicolumn{2}{|c|}{ Alcohol } & \multicolumn{2}{|c|}{ Tabaco } & \multicolumn{2}{|c|}{ Cannabis } & \multicolumn{2}{|c|}{ Felicidad } \\
\hline & & $R^{2}$ & $\beta$ & $R^{2}$ & $\beta$ & $\mathrm{R}^{2}$ & $\beta$ & $\mathrm{R}^{2}$ & $\beta$ \\
\hline Paso 1 & &, $091^{* * *}$ & &, $061^{* *}$ & & ,030* & &, 000 & \\
\hline & Sexo & &, $273^{* * *}$ & & ,078 & & ,153* & &,- 018 \\
\hline & Edad & &,$- 136^{* * *}$ & &, $233^{* *}$ & &,- 085 & & ,001 \\
\hline
\end{tabular}




\begin{tabular}{|c|c|c|c|c|c|c|c|c|c|}
\hline & & \multicolumn{2}{|c|}{ Alcohol } & \multicolumn{2}{|c|}{ Tabaco } & \multicolumn{2}{|c|}{ Cannabis } & \multicolumn{2}{|c|}{ Felicidad } \\
\hline & & $\mathrm{R}^{2}$ & $\beta$ & $\mathrm{R}^{2}$ & $\beta$ & $\mathrm{R}^{2}$ & $\beta$ & $\mathrm{R}^{2}$ & $\beta$ \\
\hline \multirow[t]{5}{*}{ Paso 2} & &, $152^{\star \star *}$ & &, $086^{* *}$ & &, $098^{* *}$ & &, 016 & \\
\hline & $\begin{array}{l}\text { Maquiavelismo } \\
\text { Manipulador }\end{array}$ & &,- 116 & &,- 055 & &,- 123 & &,- 030 \\
\hline & $\begin{array}{l}\text { Maquiavelismo } \\
\text { Estratégico }\end{array}$ & &,- 028 & &, 019 & &,- 053 & &,- 017 \\
\hline & Narcisismo & & ,139+ & &,- 008 & &, $167^{*}$ & & ,145+ \\
\hline & Psicopatía & &, $241^{* *}$ & &, $185^{*}$ & &, $243^{* *}$ & &,- 066 \\
\hline
\end{tabular}

Nota. ${ }^{* * *} p<, 001 ;{ }^{* *} p<, 01 ;{ }^{*} p<, 05 ;+p<, 10$

\section{Discusión y conclusiones}

Los resultados del estudio muestran que, de acuerdo con las hipótesis planteadas, la psicopatía es el rasgo de la triada que más se relaciona con el consumo de sustancias. Ello puede ser debido a que las personas con psicopatía destacan también por sus bajas puntuaciones en amabilidad y responsabilidad (Paulhus y Williams, 2002; Williams, Nathanson y Paulhus, 2010) si nos basamos en el modelo de los cinco factores de Costa y McCrae (Costa y McCrae, 1992). Esta combinación de rasgos están relacionados con el comportamiento antisocial y el uso y abuso de sustancias (Kotov, Gamez, Schmidt y Watson, 2010; Malouff, Thorsteinsson, Rooke y Schutte, 2007).

El narcisismo también muestra relación con el consumo de sustancias, en concreto con el consumo de alcohol y cannabis, aunque en menor grado que la psicopatía. Este resultado podría ser atribuible a la relación entre narcisimo y extraversión (Paulhus y Williams, 2002; Williams y cols., 2010; Vernon, Villani, Vickers y Harris, 2008), ya que se ha encontrado una cierta relación entre esta dimensión de personalidad y el consumo de sustancias, especialmente en adolescentes y jóvenes adultos (Ibáñez y cols., 2015).

Sin embargo, ninguno de los dos factores en los que se divide el maquiavelismo muestra relación directa con el consumo de ninguna sustancia una vez se ha controlado el efecto de la edad y el sexo, por lo que el maquiavelismo sería la dimensión de la triada oscura que menos se relaciona con el consumo de sustancias.

Respecto a la relación de la triada con el bienestar subjetivo o felicidad, el narcisismo es el único rasgo de la triada asociado a este factor, mostrando tendencia a la felicidad subjetiva. Ello estaría en consonancia con los estudios que encuentran relación del narcisismo con factores psicológicos beneficiosos como el bienestar subjetivo (Egan y cols., 2014) y la autoestima (Raskin y cols., 1991). De forma similar al consumo de alcohol, creemos que esta asociación positiva es atribuible a la relación del narcisismo con la extraversión, ya que esta dimensión de personalidad es, junto con la estabilidad emocional, el factor de personalidad más relevante para la felicidad o bienestar subjetivo (Lucas, Le y Dyrenforth, 2008; Otonari y cols., 2012; Tamir, 2009).

Por otra parte, centrándonos en los resultados de las correlaciones entre las escalas de los dos instrumentos de la triada por separado con el consumo de sustancias y felicidad, vemos que las dos escalas muestran algunas diferencias reseñables. Así, la psicopatía del SD3 se relacionaba con todas las variables de consumo, mientras que psicopatía del DD no 
muestra relación con ninguna variable de consumo de tabaco. El narcisismo muestra la misma relación con cannabis en las dos escalas, pero la escala del DD muestra también relación con variables de consumo de alcohol no obtenida por el SD3, además solo el narcisismo del SD3 muestra relación con la felicidad como se esperaba en las hipótesis. Por otra parte, el maquiavelismo solo muestra relación con el consumo de alcohol en las dos escalas, pero el maquiavlismo del DD no muestra relación con el consumo total de alcohol a la semana.

En definitiva, y a nivel de constructo, los rasgos de psicopatía, narcisismo y maquiavelismo muestran relaciones esperadas con la red nomológica, específicamente con el consumo de sustancias y el bienestar subjetivo, y ello indicaría una adecuada validez de constructo. No obstante, y a nivel de escalas específicas, se observa que el SD3 muestra mayores resultados esperados. Por lo tanto, la adaptación a nuestro contexto sociocultural del Short Dark 3 muestra mayor validez de constructo en la evaluación de la triada oscura que el Dirty Dozen.

\section{Referencias}

Chabrol, H., Van Leeuwen, N., Rodgers, R. y Séjourné, N. (2009). Contributions of psychopathic, narcissistic, machiavellian, and sadistic personality traits to juvenile delinquency. Personality and Individual Differences, 47, 734-739.

Costa, P T. y McCrae, R. R. (1992). Revised NEO Personality Inventory (NEO PI-R) and NEO Five-Factor Inventory (NEO-FFI) professional manual. Odessa, FL: Psychological Assessment Resources.

Egan, V., Chan, S. y Shorter, G. W. (2014). The Dark Triad, happiness and subjective wellbeing. Personality and Individual Differences, 67, 17-22.

Ibáñez, M. I., Camacho, L., Mezquita, L., Villa, H., Moya-Higueras, J. y Ortet, G. (2015). Alcohol expectancies mediate and moderate the associations between big five personality traits and adolescent alcohol consumption and alcohol-related problems. Frontiers in Psychology, 6, 1-8.

Jonason, P. K., Baughman, H. M., Carter, G. L. y Parker, P. (2015). Dorian Gray without his portrait: Psychological, social, and physical health costs associated with the Dark Triad. Personality and Individual Differences, 78, 5-13.

Jonason, P. K., Li, N. P. y Teicher, E. A. (2010). Who is James Bond?: The dark triad as an agentic social style. Individual Differences Research, 8, 111-120.

Jonason, P. K. y Webster, G. D. (2010). The dirty dozen: a concise measure of the dark triad. Psychological Assessment, 22, 420-432.

Jones, D. N. y Paulhus, D. L. (2014). Introducing the short Dark Triad (SD3): a brief measure of dark personality traits. Assessment, 21, 28-41.

Kotov, R., Gamez, W., Schmidt, F. y Watson, D. (2010). Linking "big" personality traits to anxiety, depressive, and substance use disorders: a meta-analysis. Psychological Bulletin, 136, 768-821.

Lucas, R. E., Le, K. y Dyrenforth, P. S. (2008). Explaining the extraversion/positive affect relation: Sociability cannot account for extraverts' greater happiness. Journal of Personality, 76, 385-414.

Lyubomirsky, S. y Lepper, H. (1999). A mesure of subjective happiness: preliminary reliability and construct validation. Social Indicators Research, 46, 137-155.

Malouff, J., Thorsteinsson, E., Rooke, S. y Schutte, N. (2007). Alcohol Involvement and the Five-Factor Model of Personality: A Meta-Analysis. Journal of Drug Education, 37, 277294. 
Nohales, B. (2015). La triada oscura de la personalidad. Adaptación al español de los cuestionarios Dirty Dozen y Short Dark Triad. Trabajo fin de grado. Universitat Jaume I, Castellón, España.

Otonari, J., Nagano, J., Morita, M., Budhathoki, S., Tashiro, N., Toyomura, K. y cols. (2012). Neuroticism and extraversion personality traits, health behaviours, and subjective wellbeing: The Fukuoka Study (Japan). Quality of Life Research, 21, 1847-1855.

Paulhus, D. L. y Williams, K. M. (2002). The Dark Triad of Personality: Narcissism, MachiaveIlianism, and Psychopathy. Journal of Research in Personality, 36, 556-563.

Raskin, R., Novacek, J. y Hogan, R. (1991). Narcissism, self-esteem, and defensive self-enhancement. Journal of Personality, 59, 19-38.

Rodríguez-Martos, A., Gual, A. y Llopis, J. J. (1999). La unidad de bebida estándar como registro simplificado del consumo de bebidas alcohólicas y su determinación en España. Medicina Clínica, 112, 446-450.

Tamir, M. (2009). Differential preferences for happiness: Extraversion and trait-consistent emotion regulation. Journal of Personality, 77, 447-470.

Vernon, P. A., Villani, V. C., Vickers, L. C. y Harris, J. A. (2008). A behavioral genetic investigation of the Dark Triad and the Big 5. Personality and Individual Differences, 44, 445452.

Williams, K. M., Nathanson, C. y Paulhus, D. L. (2010). Identifying and profiling scholastic cheaters: their personality, cognitive ability, and motivation. Journal of Experimental Psychology Applied, 16, 293-307. 